\title{
Cross-cultural adaptation of the Brazilian version of the Questionnaire on Eating and Weight Patterns-5 (QEWP-5)
}

\author{
Adaptação transcultural da versão brasileira do Questionnaire on Eating and \\ Weight Patterns-5 (QEWP-5)
}

\begin{abstract}
Carlos Eduardo Ferreira de Moraes, ${ }^{1,2}$ (D) Carla Mourilhe, ${ }^{1,2}$ Sílvia Regina de Freitas, ${ }^{2,3}$ Glória Valéria da Veiga, ${ }^{1}$
\end{abstract} Marsha D. Marcus, ${ }^{4}$ José Carlos Appolinário²

\begin{abstract}
Introduction: The Questionnaire on Eating and Weight Patterns-5 (QEWP-5) is a self-report instrument developed to screen individuals for binge eating disorder (BED), as defined by the DSM-5. However, this version of the instrument had not been adapted for the Brazilian population.

Objective: To describe translation and cross-cultural adaptation of the QEWP-5 into Brazilian Portuguese.

Methods: Translation and cross-cultural adaptation of the QEWP5 included the following steps: forward translation, comparison of translations and a synthesis version, blind back-translations, comparison of the back translations with the original version, and a comprehensibility test. The comprehensibility test was conducted with a sample of 10 participants with BED or bulimia nervosa and 10 eating disorders experts. Additionally, a Content Validity Index (CVI-I) was calculated for each item and then averaged to produce an index for the entire scale (CVI-Ave), to assess content equivalence.

Results: Some inconsistencies emerged during the process of translation and adaptation. However, the expert committee solved them by consensus. The participants of the comprehensibility test understood the Brazilian version of QEWP- 5 well. Only 2 patients $(20 \%)$ had doubts about items related to subjective binge eating episodes. Content equivalence analysis rated all items relevant, with CVI-I ranging from 0.8 to 1.0 and an overall CVI-Ave of 0.94. In view of the good overall assessment of the pre-final version of the instrument, additional changes were not made to the final version.

Conclusion: The Brazilian version of the QEWP- 5 was crossculturally adapted and was well understood by the target population. Further studies are required to assess its psychometric properties.

Keywords: Binge eating disorder, cross-cultural adaptation, QEWP-5, bulimia nervosa.
\end{abstract}

\section{Resumo}

Introdução: O Questionnaire on Eating and Weight Patterns-5 (QEWP-5) - Questionário sobre Padrões de Alimentação e Peso5 - é um instrumento auto preenchível utilizado para rastrear indivíduos com transtorno da compulsão alimentar (TCA) segundo os critérios do DSM-5. Entretanto, essa versão do instrumento ainda não foi adaptada para a população brasileira.

Objetivo: Descrever a tradução e adaptação transcultural do QEWP-5 para a língua portuguesa.

Métodos: O processo de adaptação transcultural incluiu as seguintes etapas: tradução, comparação das traduções e elaboração da versão síntese, retro-tradução com cegamento, comparação das retrotraduções com a versão original, e teste de compreensibilidade. $\mathrm{O}$ teste de compreensibilidade foi conduzido em uma amostra de 10 indivíduos com TCA ou bulimia nervosa e 10 especialistas em Transtornos Alimentares. Adicionalmente, foram calculados o Índice de Validade de Conteúdo para cada item (IVC-I) e para a média da escala (IVC-M), para avaliar a equivalência de conteúdo.

Resultados: Durante 0 processo de tradução e adaptação surgiram algumas discrepâncias. No entanto, elas foram solucionadas por meio de consenso do comitê de especialistas. No teste de compreensibilidade, a versão brasileira do QEWP-5 foi bem compreendida pelos participantes. Somente 2 participantes $(20 \%)$ apresentaram questionamentos sobre itens relacionados aos episódios de compulsão alimentar subjetivos. Em relação à equivalência de conteúdo, todos os itens foram avaliados como relevantes, com o IVC-I variando de 0,8 a 1,0. Ademais, o IVC-M foi 0,94 . Considerando a boa avaliação geral da versão pré-final do instrumento, não foram realizadas alterações na versão final. Conclusão: A versão brasileira do QEWP-5 foi adaptada transculturalmente e bem compreendida pela população-alvo. Estudos adicionais são necessários para avaliar suas propriedades psicométricas.

Descritores: TCA, adaptação transcultural, QEWP-5, bulimia nervosa.

${ }^{1}$ Departamento de Nutrição Social e Aplicada (DNSA), Instituto de Nutrição Josué de Castro (INJC), Universidade Federal do Rio de Janeiro (UFRJ), Rio de Janeiro, RJ, Brazil. ${ }^{2}$ Grupo de Obesidade e Transtornos Alimentares (GOTA), Instituto de Psiquiatria, UFRJ, Rio de Janeiro, RJ, Brazil. ${ }^{3}$ Instituto Estadual de Diabetes e Endocrinologia Luiz Capriglione (IEDE), Rio de Janeiro, RJ, Brazil. ${ }^{4}$ Department of Psychiatry, School of Medicine, University of Pittsburgh, Pittsburgh, PA, USA. This article is based on the first author's dissertation titled "Questionnaire on Eating and Weight Patterns-5 - QEWP-5 (Questionário sobre Padrões de Alimentação e Peso-5): tradução, adaptação transcultural e estudo de confiabilidade," presented at Departamento de Nutrição Social e Aplicada (DNSA), Instituto de Nutrição Josué de Castro (INJC), Universidade Federal do Rio de Janeiro (UFRJ), Rio de Janeiro, RJ, Brazil, in 2019.

Submitted Apr 02 2019, accepted for publication Jun 06 2019. Epub Mar 022020.

Suggested citation: Moraes CEF, Mourilhe C, Freitas SR, Veiga GV, Marcus MD, Appolinário JC. Cross-cultural adaptation of the Brazilian version of the Questionnaire on Eating and Weight Patterns-5 (QEWP-5). Trends Psychiatry Psychother. 2020;42(1):39-47. http://dx.doi.org/10.1590/2237-6089-2019-0029 


\section{Introduction}

Binge-eating disorder (BED) is an eating disorder recognized in the 5 th edition of the Diagnostic and Statistical Manual of Mental Disorders, (DSM-5), ${ }^{1}$ and characterized by recurrent episodes of binge eating (eating an unusually large amount of food associated with a sense of loss of control over eating). Additionally, there is marked distress related to these episodes. In BED, binge eating occurs at least once a week over a 3-month period and is not followed by the inappropriate compensatory behaviors seen in bulimia nervosa (BN). ${ }^{1}$ Additionally, binge episodes must be associated with at least 3 of the following symptoms: eating more rapidly than normal, eating until feeling uncomfortable, eating large amounts of food when not physically hungry, eating alone because of embarrassment over the amount of food being consumed, or having feelings of disgust, guilt, or depression following these episodes. BED is the most common eating disorder ${ }^{2}$ and is associated with physical, psychological, and functional impairment. ${ }^{3}$

The definition of a binge eating episode is one of the difficulties involving diagnosis of BED. Binge eating is defined as: 1 ) eating in a discrete period of time (usually less than 2 hours), a quantity of food definitely larger than most people would eat under similar circumstances; and 2) a sense of lack of control (feeling that one could not stop or control what or how much one is eating). This central component of BED diagnosis, also called objective binge eating $(O B E)$ is difficult to assess because there is no exact definition of what is considered "a quantity of food definitely larger than most people would eat," and also because the sense of lack of control is based only on one's own perception. ${ }^{1,4}$

Several instruments have been developed to assess symptoms of eating disorders and to assess binge eating. The most widely used measures include: 1) the Eating Disorders Examination - Questionnaire $(E D E-Q),{ }^{5}$ a self-report version of the EDE interview ${ }^{6}$ developed to assess the frequency and severity of eating disorder behaviors and psychopathology; 2) the Binge Eating Scale (BES), ${ }^{7}$ developed to assess binge eating severity in individuals with obesity (The BES has been adapted for Portuguese ${ }^{8}$ and validated in obese Brazilian women) ${ }^{9}$; and 3 ) the Questionnaire on Eating and Weight Patterns-Revised (QEWP-R), ${ }^{10}$ which is designed to screen individuals for BED, as diagnosed by the DSM-IV. ${ }^{11}$ The QEWP-R has been adapted and validated for the Brazilian population. ${ }^{12}$

As a result of changes made in the DSM- 5 , instruments developed to assess BED according to previous criteria needed to be updated in line with the current diagnostic criteria. The QEWP-R was therefore updated as the
QEWP-5, ${ }^{13}$ a 26-item questionnaire that includes the following modifications: 1 ) revision of the frequency of binge eating and compensatory behaviors; 2) revision of the threshold for inappropriate compensatory behaviors - exclusion criteria; 3) removal of some questions that were not related to the diagnostic criteria; 4) incorporation of questions to assess subjective binge eating - SBE (loss of control eating in the absence of consuming a large quantity of food); and 5) revision of the decision rules for diagnosis. ${ }^{13}$

However, to date, the QEWP-5 has not been translated into or adapted for Portuguese. Cross-cultural adaptation of the QEWP-5, following international guidelines, is therefore essential to make available a correctly translated instrument for use in Brazilian settings.

The present study aims to describe the process of cross-cultural adaptation of the QEWP-5 for Brazilian Portuguese.

\section{Methods}

Permission to cross-culturally adapt the scale for Brazilian Portuguese was requested from and granted by the original authors of the QEWP-5. We began a process of symmetrical translation based on the stages proposed by Sousa \& Rojjanasrirat. ${ }^{14}$ This methodology involves the following five steps:

\section{Forward translation}

Forward translations were conducted by two independent bilingual eating disorder specialists ( $\mathrm{T} 1$ and T2), whose native language is Brazilian Portuguese. They produced two versions ( $\mathrm{T} 1$ and $\mathrm{T} 2$ ) of the instrument.

\section{Comparison of the translations and synthesis version}

A third eating disorder specialist with experience in translation, adaptation, and validation of scales compared the two different translations ( $\mathrm{T} 1$ and $\mathrm{T} 2$ ) with the original version of the QEWP-5 and evaluated any semantic inconsistencies (including any linguistic or conceptual issues). After these comparisons, a merged and synthesized version of the two translations was produced (SV).

The three translated versions ( $\mathrm{T} 1, \mathrm{~T} 2$ and $\mathrm{SV}$ ) were presented to an eating disorders expert committee (three psychiatrists, one dietitian and one psychologist). Ambiguities and discrepancies were discussed, and consensus was achieved, with participation of all three translators. This process generated the preliminary version (PV) of the translated instrument. 


\section{Blinded back-translation}

The PV was back translated into English by two other independent translators whose native language was English, but who had different profiles. The first was experienced in psychiatric terminology and the second translator was more familiar with colloquial phrases and emotional terms in English. They were blinded to the original version of the QEWP-5. This process resulted in two back-translated versions (BTL-1 and BTL-2) of the instrument.

\section{Comparison of the back-translations}

The two back-translations were compared with the original instrument. One of the developers of the original version of the QEWP-5 participated in this step, evaluating both BTL-1 and BTL-2. This step generated the pre-final version (PFV) of the QEWP-5 in Brazilian Portuguese.

\section{Comprehensibility}

The PFV was tested on 20 participants (10 patients and 10 experts) as proposed by Sousa and Rojjanasrirat. ${ }^{14}$

The comprehensibility of the PFV was pilot tested with 10 participants recruited from the Obesity and Eating Disorders Group (Universidade Federal do Rio de Janeiro), previously diagnosed with BED and BN according to the DSM-5 diagnostic criteria. Their native language was Brazilian Portuguese. They all read, answered and rated a form containing questions about the comprehensibility of the items using a dichotomous scale (clear or unclear). They were also asked to provide suggestions for items they rated as unclear. Items rated unclear by at least $20 \%$ of the participants were revised.

Next, a group of 10 eating disorder experts (who were not on the initial expert committee) were invited to evaluate the comprehensibility and relevance of the items on the scale. First, each expert rated the items as clear or unclear, and provided suggestions to make the language clearer. They then evaluated content equivalence using the following ratings: 1 ) not relevant; 2) unable to assess relevance; 3) relevant but needs minor alteration; 4) very relevant and succinct. ${ }^{15}$ Items rated as unclear by at least $20 \%$ of the experts (comprehensibility evaluation) and classified as 1 or 2 on the relevance scale were revised. Finally, a Content Validity Index (CVI) was calculated for each item (CVI-I) and then averaged to produce an index for the entire scale (CVI-Ave). The minimum cutoffs for acceptability were an individual CVI-I of 0.78 or above ${ }^{15}$ and a CVIAve of 0.90 or above. ${ }^{16}$

This study was approved by the ethics committee at Instituto de Psiquiatria, Universidade Federal do Rio de
Janeiro (UFRJ), Rio de Janeiro, Brazil. Written informed consent was obtained from all study participants before any of the study procedures were performed.

\section{Results}

\section{Translation, cross-cultural adaptation}

Certain semantic inconsistencies emerged during the process of Portuguese translation and cultural adaptation of the QEWP-5. The three translators (T1, T2, and SV) made a special effort to use colloquial expressions/ phrases to make the scale easily understood by the target population. Although they produced quite similar translations, there were ambiguities in certain items. Table 1 shows comparisons between T1, T2, SV, and the original version of QEWP-5 for the most debated items.

With relation to item 9, for example, the translators disagreed on how to translate the expression "during the times" (in Portuguese "nas ocasiões" or "nas vezes"). In the final version, the final consensus was to use "nas vezes." In items 11 and 23, the expression "feeling disgusted with yourself" was initially translated as "sentir repugnância por si mesmo." However, in the SV this expression was changed to "sentir repulsa por si mesmo," because the Portuguese version of DSM-5 uses the word "repulsa" to describe one of the symptoms associated with binge eating.

There was also disagreement on how to translate to the word "upset" in items 13 and 25. The Portuguese word chosen in the final version was "perturbaram," because it was considered that this expression best describes the distress associated with binge eating. Item 26 about parents' silhouettes was the subject of some debate. The consensus was that the Portuguese translations used in T1 and T2 versions for "If you have no knowledge of your biological father and/or mother, don't circle anything for that" was difficult to understand (in Portuguese, "Se você não conhece seu pai e/ou sua mãe biológicos não circule aquele que não conhece"). In the SV, this sentence was therefore changed for the following Portuguese expression "se você não conhece seu pai e/ou mãe biológicos, não circule nada para esse pai e/ou mãe, isto é, circule apenas para o pai e/ou mãe biológicos que você conhece." However, since the silhouettes were introduced in QEWP-5 for research purposes only and are not a diagnostic item, they can be omitted without prejudice.

An expert committee evaluated and compared the SV with the original version of QEWP-5. This group suggested some changes to address inconsistencies. Table 2 shows a summary of the items modified after the expert panel and 
Table 1 - Comparison of the original version, translation 1, translation 2, and the synthesis version of the QEWP-5 (items 9, 11, 13, 23 , and 26).

\begin{tabular}{|c|c|c|c|c|}
\hline Item & Original version & Translation 1 & Translation 2 & Synthesis version \\
\hline 9 & $\begin{array}{l}\text { During the times when you ate an } \\
\text { unusually large amount of food, did } \\
\text { you ever feel you could not stop } \\
\text { eating or control what or how much } \\
\text { you were eating? }\end{array}$ & $\begin{array}{l}\text { Nas ocasiões em que você comeu } \\
\text { quantidades de comida que a } \\
\text { maioria das pessoas consideraria } \\
\text { demais, você sentiu que não } \\
\text { conseguia parar de comer ou } \\
\text { controlar o que ou quanto comia? }\end{array}$ & $\begin{array}{l}\text { Nas ocasiões em que você comeu } \\
\text { quantidades de comida que a } \\
\text { maioria das pessoas consideraria } \\
\text { demais, você sentiu que não } \\
\text { conseguia parar de comer ou } \\
\text { controlar o que ou quanto comia? }\end{array}$ & $\begin{array}{l}\text { Nas vezes em que você comeu uma } \\
\text { quantidade excepcionalmente grande } \\
\text { de comida, você sempre sentia que } \\
\text { não poderia parar de comer, nem } \\
\text { controlar o que e o quanto você } \\
\text { estava comendo? }\end{array}$ \\
\hline $\begin{array}{l}11 \mathrm{e} \\
23 \mathrm{e}\end{array}$ & $\begin{array}{l}\text { Feeling disgusted with yourself, } \\
\text { depressed, or feeling very guilty } \\
\text { afterward? }\end{array}$ & $\begin{array}{l}\text { Sentia repugnância por você } \\
\text { mesmo, muita culpa ou depressão } \\
\text { após o episódio de excesso } \\
\text { alimentar? }\end{array}$ & $\begin{array}{l}\text { Sentir repugnância por si mesmo, } \\
\text { muito culpado ou deprimido logo } \\
\text { após o episódio }\end{array}$ & $\begin{array}{l}\text { Sentir-se desgostoso consigo mesmo, } \\
\text { deprimido ou muito culpado depois } \\
\text { do episódio? }\end{array}$ \\
\hline 13 & $\begin{array}{l}\text { In general, during the past three } \\
\text { months, how upset were you by } \\
\text { these episodes (when you ate a } \\
\text { large amount of food and felt your } \\
\text { eating was out of control)? }\end{array}$ & $\begin{array}{l}\text { De modo geral, nos últimos três } \\
\text { meses, quanto você se aborreceu } \\
\text { por causa desses episódios (quando } \\
\text { você comeu grandes quantidades de } \\
\text { comida e sentiu que não conseguia } \\
\text { parar de comer ou controlar o que/ } \\
\text { quanto comia)? }\end{array}$ & $\begin{array}{l}\text { De modo geral, nos últimos três } \\
\text { meses, quanto você se aborreceu } \\
\text { por causa destes episódios (em } \\
\text { que comeu grandes quantidades } \\
\text { de comida e sentiu que a sua } \\
\text { alimentação estava fora de } \\
\text { controle)? }\end{array}$ & $\begin{array}{l}\text { Em geral, durante os últimos três } \\
\text { meses, quanto esses episódios } \\
\text { perturbaram você (os episódios } \\
\text { em que você comeu uma grande } \\
\text { quantidade de comida e sentiu que } \\
\text { a sua alimentação estava fora do } \\
\text { controle)? }\end{array}$ \\
\hline 26 & $\begin{array}{l}\text { Please take a look at these } \\
\text { silhouettes. Put a circle around the } \\
\text { silhouettes that most resemble the } \\
\text { body builds of your biological father } \\
\text { and mother at their heaviest. If you } \\
\text { have no knowledge of your biological } \\
\text { father and/or mother, don't circle } \\
\text { anything for that }\end{array}$ & $\begin{array}{l}\text { Por favor, observe essas silhuetas. } \\
\text { Circule aquela que mais se } \\
\text { assemelha ao feitio do corpo de seu } \\
\text { pai e/ou sua mãe }\end{array}$ & $\begin{array}{l}\text { Por favor, observe essas silhuetas. } \\
\text { Circule aquela que mais se } \\
\text { assemelha ao feitio do corpo de seu } \\
\text { pai e sua mãe biológicos no seu } \\
\text { peso mais alto. Se você não conhece } \\
\text { seu pai e/ou mãe biológicos, não } \\
\text { circule aquele que conhece. }\end{array}$ & $\begin{array}{l}\text { Por favor, observe essas silhuetas. } \\
\text { Circule aquela que mais se } \\
\text { assemelha ao feitio do corpo de seu } \\
\text { pai e sua mãe biológicos no seu peso } \\
\text { mais alto. Se você não conhece seu } \\
\text { pai e/ou mãe biológicos, não circule } \\
\text { nada para esse pai e/ou mãe, isto é, } \\
\text { circule apenas para o pai e/ou mãe } \\
\text { biológicos que você conhece. }\end{array}$ \\
\hline
\end{tabular}

Table 2 - Examples of items changed after the panel of experts: Comparison of the original version, synthesis version (SV), preliminary version (PV), back-translations (BTL-1 and BTL-2) and the final version (FV) of the QEWP-5

\begin{tabular}{|c|c|c|c|c|c|c|}
\hline Item & Original version & sv & PV & BTL-1 & BTL-2 & FV \\
\hline 9 & $\begin{array}{l}\text { During the times when } \\
\text { you ate an unusually } \\
\text { large amount of food, } \\
\text { did you ever feel you } \\
\text { could not stop eating } \\
\text { or control what or how } \\
\text { much you were eating? }\end{array}$ & $\begin{array}{l}\text { Nas vezes em que você } \\
\text { comeu uma quantidade } \\
\text { excepcionalmente } \\
\text { grande de comida, você } \\
\text { sempre sentia que não } \\
\text { poderia parar de comer, } \\
\text { nem controlar o que e } \\
\text { o quanto você estava } \\
\text { comendo? }\end{array}$ & $\begin{array}{l}\text { Nas vezes em que você } \\
\text { comeu uma quantidade } \\
\text { excepcionalmente } \\
\text { grande de comida, você } \\
\text { alguma vez sentiu que } \\
\text { não poderia parar de } \\
\text { comer ou controlar o } \\
\text { que ou o quanto você } \\
\text { estava comendo? }\end{array}$ & $\begin{array}{l}\text { The times you ate a } \\
\text { quantity exceptionally } \\
\text { large, did you ever feel } \\
\text { that you could not stop } \\
\text { eating or control what } \\
\text { or how much you were } \\
\text { eating? }\end{array}$ & $\begin{array}{l}\text { When you have eaten } \\
\text { an unusually large } \\
\text { amount of food, have } \\
\text { you ever felt that you } \\
\text { couldn't stop eating or } \\
\text { control what or how } \\
\text { much you were eating? }\end{array}$ & $\begin{array}{l}\text { Nas vezes em que você } \\
\text { comeu uma quantidade } \\
\text { excepcionalmente } \\
\text { grande de comida, você } \\
\text { alguma vez sentiu que } \\
\text { não poderia parar de } \\
\text { comer ou controlar o que } \\
\text { ou o quanto você estava } \\
\text { comendo? }\end{array}$ \\
\hline 10 & $\begin{array}{l}\text { During the past three } \\
\text { months, how often, } \\
\text { on average, did you } \\
\text { have episodes like } \\
\text { this -- that is, eating } \\
\text { large amounts of food } \\
\text { plus the feeling that } \\
\text { your eating was out of } \\
\text { control? (There may } \\
\text { have been some weeks } \\
\text { when this did not } \\
\text { happen -- just average } \\
\text { those in.) }\end{array}$ & $\begin{array}{l}\text { Durante os últimos } \\
\text { três meses, com que } \\
\text { frequência, em média, } \\
\text { você teve episódios } \\
\text { como esse - isto é, } \\
\text { episódios em que comia } \\
\text { grandes quantidades } \\
\text { de comida com a } \\
\text { sensação de que sua } \\
\text { alimentação estava } \\
\text { fora do controle? (Pode } \\
\text { ter havido algumas } \\
\text { semanas em que isto } \\
\text { não aconteceu - calcule } \\
\text { a média..). }\end{array}$ & $\begin{array}{l}\text { Durante os últimos } \\
\text { três meses, com } \\
\text { que frequência, em } \\
\text { média, você teve } \\
\text { episódios como esse } \\
\text { - isto é, episódios em } \\
\text { que comeu grandes } \\
\text { quantidades de comida } \\
\text { acompanhado da } \\
\text { sensação de que sua } \\
\text { alimentação estava } \\
\text { fora do controle? (Pode } \\
\text { ter havido algumas } \\
\text { semanas em que isto } \\
\text { não aconteceu - dê } \\
\text { uma média). }\end{array}$ & $\begin{array}{l}\text { During the last three } \\
\text { months, how often, on } \\
\text { average, did you have } \\
\text { episodes like this - that } \\
\text { is, episodes when you } \\
\text { ate large quantities of } \\
\text { food accompanied } \\
\text { by the sensation that } \\
\text { your eating was out } \\
\text { of control? (There } \\
\text { could have been some } \\
\text { weeks when this did } \\
\text { not happen - give an } \\
\text { average). }\end{array}$ & $\begin{array}{l}\text { In the last three } \\
\text { months, how often on } \\
\text { average did you have } \\
\text { episodes like this - in } \\
\text { which you ate large } \\
\text { amounts of food along } \\
\text { with the feeling that } \\
\text { your eating was out of } \\
\text { control? (There may } \\
\text { have been some weeks } \\
\text { when this didn't happen } \\
\text { - give an average). }\end{array}$ & $\begin{array}{l}\text { Durante os últimos } \\
\text { três meses, com que } \\
\text { frequência, em média, } \\
\text { você teve episódios } \\
\text { como esse - isto é, } \\
\text { episódios em que comeu } \\
\text { grandes quantidades de } \\
\text { comida acompanhado } \\
\text { da sensação de que } \\
\text { sua alimentação estava } \\
\text { fora do controle? (Pode } \\
\text { ter havido algumas } \\
\text { semanas em que isto } \\
\text { não aconteceu - dê uma } \\
\text { média). }\end{array}$ \\
\hline $\begin{array}{l}12 c \\
24 c\end{array}$ & $\begin{array}{l}\text { As best you can } \\
\text { remember, please list } \\
\text { everything you ate } \\
\text { and drank during that } \\
\text { episode. Please list the } \\
\text { foods eaten and liquids } \\
\text { consumed during the } \\
\text { episode. Be specific - } \\
\text { include brand names } \\
\text { where possible, and } \\
\text { amounts or portion } \\
\text { sizes as best you can } \\
\text { estimate. }\end{array}$ & $\begin{array}{l}\text { Por favor, procure se } \\
\text { lembrar da melhor } \\
\text { forma possível e } \\
\text { escreva abaixo tudo } \\
\text { o que você comeu e } \\
\text { bebeu durante esse } \\
\text { episódio. Por favor, faça } \\
\text { uma lista dos alimentos } \\
\text { ingeridos e dos líquidos } \\
\text { consumidos durante } \\
\text { o episódio. Seja } \\
\text { detalhista - inclua os } \\
\text { nomes das marcas } \\
\text { onde for possível e } \\
\text { estime as quantidades } \\
\text { ou o tamanho das } \\
\text { porções com a máxima } \\
\text { precisão que puder }\end{array}$ & $\begin{array}{l}\text { Por favor, procure se } \\
\text { lembrar da melhor } \\
\text { forma possível e } \\
\text { escreva abaixo tudo } \\
\text { o que você comeu e } \\
\text { bebeu durante esse } \\
\text { episódio. Por favor, faça } \\
\text { uma lista dos alimentos } \\
\text { ingeridos e dos líquidos } \\
\text { consumidos durante } \\
\text { o episódio. Seja } \\
\text { detalhista - inclua os } \\
\text { nomes das marcas } \\
\text { onde for possível e } \\
\text { estime as quantidades } \\
\text { ou o tamanho das } \\
\text { porções com a maior } \\
\text { precisão que puder. }\end{array}$ & $\begin{array}{l}\text { Please try to remember } \\
\text { as best as possible and } \\
\text { write below everything } \\
\text { you ate and drank } \\
\text { during this episode. } \\
\text { Please make a list of } \\
\text { foodstuffs ingested } \\
\text { and liquids consumed } \\
\text { during the episode. Be } \\
\text { detailed - include the } \\
\text { names of the brands } \\
\text { when possible and } \\
\text { estimate the quantities } \\
\text { or portion sizes with } \\
\text { the highest precision } \\
\text { you can. }\end{array}$ & $\begin{array}{l}\text { Please try to remember } \\
\text { as best as you can } \\
\text { and write below what } \\
\text { you ate and drank } \\
\text { during this episode. } \\
\text { Please make a list of } \\
\text { the foods you ate and } \\
\text { the liquids you drank } \\
\text { during the episode. Be } \\
\text { detailed - include the } \\
\text { brand names whenever } \\
\text { possible and estimate } \\
\text { the amounts or size } \\
\text { of the servings as } \\
\text { accurately as you can. }\end{array}$ & $\begin{array}{l}\text { Por favor, procure se } \\
\text { lembrar da melhor forma } \\
\text { possível e escreva abaixo } \\
\text { tudo o que você comeu } \\
\text { e bebeu durante esse } \\
\text { episódio. Por favor, faça } \\
\text { uma lista dos alimentos } \\
\text { ingeridos e dos líquidos } \\
\text { consumidos durante o } \\
\text { episódio. Seja detalhista } \\
\text { - inclua os nomes das } \\
\text { marcas onde for possível } \\
\text { e estime as quantidades } \\
\text { ou o tamanho das } \\
\text { porções com a maior } \\
\text { precisão que puder. }\end{array}$ \\
\hline
\end{tabular}


the respective comparisons between the original version, $\mathrm{SV}, \mathrm{PV}$, the back-translations and the final version of the QEWP-5. Ambiguities affecting items 10, 12c, 22, and 24c were solved. For items 10 and 22 (about frequency of OBE and SBE, respectively), the expression "just average those in" was translated to "dê uma média" in the PV.
There was consensus in the committee that questions $12 \mathrm{c}$ and $24 \mathrm{c}$ (describing a typical binge eating episode) should not suggest examples of brands or quantities of foods, to avoid inducing answers.

After this step, some additional suggestions made by the eating disorder experts were incorporated into

Table 2 (cont.)

\begin{tabular}{|c|c|c|c|c|c|c|}
\hline Item & Original version & sv & PV & BTL-1 & BTL-2 & $\mathbf{F V}$ \\
\hline 18 & $\begin{array}{l}\text { During the past three } \\
\text { months, did you ever } \\
\text { exercise excessively } \\
\text {-for example, } \\
\text { exercised even though } \\
\text { it interfered with } \\
\text { important activities or } \\
\text { despite being injured } \\
\text {-specifically in order } \\
\text { to avoid gaining weight } \\
\text { after episodes of eating } \\
\text { like you described } \\
\text { (when you ate a large } \\
\text { amount of food and felt } \\
\text { your eating was out of } \\
\text { control)? }\end{array}$ & $\begin{array}{l}\text { Durante os últimos três } \\
\text { meses, você alguma } \\
\text { vez se exercitou } \\
\text { excessivamente - } \\
\text { por exemplo, fez } \\
\text { exercícios mesmo } \\
\text { quando eles interferiam } \\
\text { em importantes } \\
\text { atividades ou mesmo } \\
\text { estando machucado } \\
\text { - especificamente } \\
\text { para evitar ganhar peso } \\
\text { após ter tido episódios } \\
\text { como esses que } \\
\text { você descreveu (em } \\
\text { que comeu grandes } \\
\text { quantidades de comida } \\
\text { e sentiu que a sua } \\
\text { alimentação estava fora } \\
\text { de controle)? }\end{array}$ & $\begin{array}{l}\text { Durante os últimos três } \\
\text { meses, você alguma } \\
\text { vez se exercitou } \\
\text { excessivamente - por } \\
\text { exemplo, fez exercícios } \\
\text { mesmo quando } \\
\text { eles interferiam em } \\
\text { atividades importantes } \\
\text { ou mesmo estando } \\
\text { machucado - } \\
\text { especificamente para } \\
\text { evitar ganhar peso } \\
\text { após ter tido episódios } \\
\text { como esses que } \\
\text { você descreveu (em } \\
\text { que comeu grandes } \\
\text { quantidades de comida } \\
\text { e sentiu que a sua } \\
\text { alimentação estava fora } \\
\text { de controle)? }\end{array}$ & $\begin{array}{l}\text { During the last three } \\
\text { months, did you ever } \\
\text { exercise excessively } \\
\text { - for example, you } \\
\text { exercised even when } \\
\text { it interfered with } \\
\text { important activities } \\
\text { or while injured - } \\
\text { specifically to avoid } \\
\text { gaining weight after } \\
\text { having had episodes } \\
\text { like those that you } \\
\text { described (when you } \\
\text { ate a large quantity } \\
\text { of food and felt that } \\
\text { your eating was out of } \\
\text { control)? }\end{array}$ & $\begin{array}{l}\text { In the last three } \\
\text { months, have you ever } \\
\text { over-exercised - for } \\
\text { example, exercised } \\
\text { even when it interfered } \\
\text { in important activities } \\
\text { or even when you were } \\
\text { hurt - specifically to } \\
\text { avoid gaining weight } \\
\text { after having episodes } \\
\text { like the ones you } \\
\text { described (in which } \\
\text { you ate a large amount } \\
\text { of food and felt that } \\
\text { your eating was out of } \\
\text { control)? }\end{array}$ & $\begin{array}{l}\text { Durante os últimos } \\
\text { três meses, você } \\
\text { alguma vez se exercitou } \\
\text { excessivamente - } \\
\text { por exemplo, fez } \\
\text { exercícios mesmo } \\
\text { quando eles interferiam } \\
\text { em atividades } \\
\text { importantes ou mesmo } \\
\text { estando machucado } \\
\text { - especificamente } \\
\text { para evitar ganhar } \\
\text { peso após ter tido } \\
\text { episódios como esses } \\
\text { que você descreveu } \\
\text { (em que comeu grandes } \\
\text { quantidades de comida } \\
\text { e sentiu que a sua } \\
\text { alimentação estava fora } \\
\text { de controle)? }\end{array}$ \\
\hline 21 & $\begin{array}{l}\text { During the last three } \\
\text { months, did you ever } \\
\text { have episodes when } \\
\text { you felt you could not } \\
\text { stop eating or control } \\
\text { what or how much } \\
\text { you were eating, but } \\
\text { you did not consume } \\
\text { a quantity of food } \\
\text { which the majority of } \\
\text { people would consider } \\
\text { exceptionally large? }\end{array}$ & $\begin{array}{l}\text { Durante os últimos } \\
\text { três meses, algumas } \\
\text { vezes, você teve } \\
\text { episódios durante os } \\
\text { quais você sentiu que } \\
\text { não poderia parar de } \\
\text { comer, nem controlar } \\
\text { o que ou quanto você } \\
\text { estava comendo, } \\
\text { mas nos quais você } \\
\text { não consumiu uma } \\
\text { quantidade de comida } \\
\text { que a maioria das } \\
\text { pessoas consideraria } \\
\text { excepcionalmente } \\
\text { grande? }\end{array}$ & $\begin{array}{l}\text { Durante os últimos } \\
\text { três meses, alguma } \\
\text { vez você teve episódios } \\
\text { durante os quais } \\
\text { você sentiu que não } \\
\text { poderia parar de } \\
\text { comer ou controlar } \\
\text { o que ou o quanto } \\
\text { você estava comendo, } \\
\text { mas nos quais você } \\
\text { não consumiu uma } \\
\text { quantidade de comida } \\
\text { que a maioria das } \\
\text { pessoas consideraria } \\
\text { excepcionalmente } \\
\text { grande? }\end{array}$ & $\begin{array}{l}\text { During the last three } \\
\text { months, did you ever } \\
\text { have episodes when } \\
\text { you felt you could not } \\
\text { stop eating or control } \\
\text { what or how much } \\
\text { you were eating, but } \\
\text { you did not consume } \\
\text { a quantity of food } \\
\text { which the majority of } \\
\text { people would consider } \\
\text { exceptionally large? }\end{array}$ & $\begin{array}{l}\text { In last three months, } \\
\text { have you had episodes } \\
\text { in which you felt you } \\
\text { couldn't stop eating } \\
\text { or controlling what or } \\
\text { how much you were } \\
\text { eating, but you did not } \\
\text { consume an amount } \\
\text { of food that most } \\
\text { people would consider } \\
\text { unusually large? }\end{array}$ & $\begin{array}{l}\text { Durante os últimos três } \\
\text { meses, alguma vez você } \\
\text { teve episódios durante } \\
\text { os quais você sentiu que } \\
\text { não poderia parar de } \\
\text { comer ou controlar o que } \\
\text { ou o quanto você estava } \\
\text { comendo, mas nos quais } \\
\text { você não consumiu } \\
\text { uma quantidade de } \\
\text { comida que a maioria } \\
\text { das pessoas consideraria } \\
\text { excepcionalmente } \\
\text { grande? }\end{array}$ \\
\hline 22 & $\begin{array}{l}\text { During the past three } \\
\text { months how often did } \\
\text { you have episodes like } \\
\text { this -- the feeling that } \\
\text { your eating was out } \\
\text { of control, but you did } \\
\text { not consume what } \\
\text { most people would } \\
\text { think was an unusually } \\
\text { large amount of food? } \\
\text { (There may have been } \\
\text { some weeks when this } \\
\text { did not happen --just } \\
\text { average those in.) }\end{array}$ & $\begin{array}{l}\text { Durante os últimos } \\
\text { três meses, com que } \\
\text { frequência você teve } \\
\text { episódios como esse } \\
\text { - sentiu que a sua } \\
\text { alimentação estava fora } \\
\text { de controle, mas você } \\
\text { não consumiu uma } \\
\text { quantidade de comida } \\
\text { que a maioria das } \\
\text { pessoas consideraria } \\
\text { excepcionalmente } \\
\text { grande? (Pode ter } \\
\text { havido algumas } \\
\text { semanas em que isto } \\
\text { não aconteceu - calcule } \\
\text { a média...). }\end{array}$ & $\begin{array}{l}\text { Durante os últimos } \\
\text { três meses, com que } \\
\text { frequência você teve } \\
\text { episódios como esse } \\
\text { - sentiu que a sua } \\
\text { alimentação estava fora } \\
\text { de controle, mas você } \\
\text { não consumiu uma } \\
\text { quantidade de comida } \\
\text { que a maioria das } \\
\text { pessoas consideraria } \\
\text { excepcionalmente } \\
\text { grande? (Pode ter } \\
\text { havido algumas } \\
\text { semanas em que isto } \\
\text { não aconteceu - dê } \\
\text { uma média). }\end{array}$ & $\begin{array}{l}\text { During the last three } \\
\text { months, how often } \\
\text { did you have episodes } \\
\text { like this - you felt } \\
\text { that your eating was } \\
\text { out of control, but } \\
\text { you did not consume } \\
\text { a quantity of food } \\
\text { which the majority of } \\
\text { people would consider } \\
\text { exceptionally large? } \\
\text { (There could have been } \\
\text { some weeks when this } \\
\text { did not happen - give } \\
\text { an average). }\end{array}$ & $\begin{array}{l}\text { In the last three } \\
\text { months, how often } \\
\text { have you had episodes } \\
\text { like this - when you } \\
\text { felt your eating was } \\
\text { out of control, but you } \\
\text { did not consume an } \\
\text { amount of food that } \\
\text { most people would } \\
\text { consider unusually } \\
\text { large? (There may have } \\
\text { been weeks when this } \\
\text { did not happen- give } \\
\text { an average). }\end{array}$ & $\begin{array}{l}\text { Durante os últimos } \\
\text { três meses, com que } \\
\text { frequência você teve } \\
\text { episódios como esse } \\
\text { - sentiu que a sua } \\
\text { alimentação estava fora } \\
\text { de controle, mas você } \\
\text { não consumiu uma } \\
\text { quantidade de comida } \\
\text { que a maioria das } \\
\text { pessoas consideraria } \\
\text { excepcionalmente } \\
\text { grande? (Pode ter havido } \\
\text { algumas semanas em } \\
\text { que isto não aconteceu - } \\
\text { dê uma média). }\end{array}$ \\
\hline 25 & $\begin{array}{l}\text { In general, during the } \\
\text { past three months, } \\
\text { how upset were you } \\
\text { by these episodes (that } \\
\text { is, when you felt you } \\
\text { could not stop eating } \\
\text { or control what or } \\
\text { how much you were } \\
\text { eating but in which you } \\
\text { did not consume an } \\
\text { unusually large amount } \\
\text { of food)? }\end{array}$ & $\begin{array}{l}\text { De modo geral, nos } \\
\text { últimos três meses, } \\
\text { quanto você se } \\
\text { aborreceu por causa } \\
\text { destes episódios (em } \\
\text { que sentiu que não } \\
\text { poderia parar de } \\
\text { comer ou controlar o } \\
\text { que, ou como estava } \\
\text { comendo, entretanto } \\
\text { nestes episódios você } \\
\text { não consumiu uma } \\
\text { quantidade de comida } \\
\text { que a maioria das } \\
\text { pessoas consideraria } \\
\text { excepcionalmente } \\
\text { grande)? }\end{array}$ & $\begin{array}{l}\text { Em geral, durante os } \\
\text { últimos três meses, } \\
\text { quanto esses episódios } \\
\text { chatearam você } \\
\text { (os episódios em } \\
\text { que você sentiu que } \\
\text { não poderia parar de } \\
\text { comer ou controlar } \\
\text { o que ou o quanto } \\
\text { você estava comendo, } \\
\text { mas no qual você } \\
\text { não consumiu uma } \\
\text { quantidade de comida } \\
\text { excepcionalmente } \\
\text { grande)? }\end{array}$ & $\begin{array}{l}\text { In general, during the } \\
\text { last three months, } \\
\text { how much did these } \\
\text { episodes bother you } \\
\text { (episodes when you } \\
\text { felt you could not } \\
\text { stop eating or control } \\
\text { what or how much } \\
\text { you were eating, but } \\
\text { you did not consume } \\
\text { an exceptionally large } \\
\text { quantity of food)? }\end{array}$ & $\begin{array}{l}\text { In general, in the } \\
\text { last three months, } \\
\text { how much did these } \\
\text { episodes bother you } \\
\text { (the episodes in which } \\
\text { felt you could not stop } \\
\text { eating or control what } \\
\text { or how much you were } \\
\text { eating, but you did not } \\
\text { consume an usually } \\
\text { large amount of food)? }\end{array}$ & $\begin{array}{l}\text { Em geral, durante os } \\
\text { últimos três meses, } \\
\text { quanto esses episódios } \\
\text { chatearam você (os } \\
\text { episódios em que você } \\
\text { sentiu que não poderia } \\
\text { parar de comer ou } \\
\text { controlar o que ou o } \\
\text { quanto você estava } \\
\text { comendo, mas no qual } \\
\text { você não consumiu uma } \\
\text { quantidade de comida } \\
\text { excepcionalmente } \\
\text { grande)? }\end{array}$ \\
\hline
\end{tabular}


to the SV, resulting in the PV. This version was back translated to English. BTL-1 and BTL-2 were sent to the author of the original version of QEWP-5. Additionally, detailed explanations of each change made to the Portuguese version of the instrument were provided. All alterations were approved by the author. The PFV was then generated and the pilot test was conducted.

\section{Comprehensibility}

The PFV was pilot tested on ten patients (two men and eight women) diagnosed with BED $(n=7)$ and BN $(n=3)$, to evaluate the instructions and the items and to ask them questions regarding the comprehensibility of the QEWP-5. The participants had a mean age of 37.5 years $(S D=10.5)$ and $80 \%$ of them had a college degree. Table 3 shows percentage comprehension ratings from the patients and eating disorder experts for the PFV.

The Brazilian version of the QEWP-5 was well understood by the patients. The main doubts were related to the expression defining SBE (questions 21,
23, and 24). In item 21, one participant considered the expression in Portuguese "mas nos quais" very formal and difficult to understand. In addition, in item 23, one participant questioned the consistency of sub-items b (in Portuguese, "Comer até se sentir desconfortavelmente cheio") and c (in Portuguese, "Comer grandes quantidades de comida sem estar fisicamente com fome"). The patient pointed out that if the item were related to SBE (when there is a sense of loss of control without consuming a large amount of food), these two sub-items did not make sense because they are related to objective binge eating (consider the ingestion of a large quantity of food). Finally, in item 24 (about the characteristics of a typical SBE episode), one participant asked if the question was similar to item 12 (about the characteristics of a typical OBE episode). Also, two patients asked if they could describe more than one episode of SBE.

A group of ten experts in eating disorders (five psychiatrists, two nutritionists, two psychologists, and one nurse) was invited to evaluate the instructions,

Table 3 - Comprehensibility (patients and eating disorder experts) and relevance (eating disorder experts) of items in the Brazilian version of QEWP-5

\begin{tabular}{|c|c|c|c|c|c|}
\hline \multirow{2}{*}{ Item } & \multicolumn{2}{|c|}{ Patients } & \multicolumn{3}{|c|}{ Eating disorder experts } \\
\hline & Clear & Unclear & Clear & Unclear & CVI-I \\
\hline 1 & $100 \%$ & $0 \%$ & $100 \%$ & $0 \%$ & 0.9 \\
\hline 2 & $100 \%$ & $0 \%$ & $100 \%$ & $0 \%$ & 0.9 \\
\hline 3 & $100 \%$ & $0 \%$ & $100 \%$ & $0 \%$ & 0.9 \\
\hline 4 & $100 \%$ & $0 \%$ & $80 \%$ & $20 \%$ & 0.9 \\
\hline 5 & $100 \%$ & $0 \%$ & $90 \%$ & $10 \%$ & 0.9 \\
\hline 6 & $100 \%$ & $0 \%$ & $100 \%$ & $0 \%$ & 0.9 \\
\hline 7 & $100 \%$ & $0 \%$ & $100 \%$ & $0 \%$ & 0.9 \\
\hline 8 & $80 \%$ & $20 \%$ & $90 \%$ & $10 \%$ & 1 \\
\hline 9 & $90 \%$ & $10 \%$ & $100 \%$ & $0 \%$ & 1 \\
\hline 10 & $100 \%$ & $0,00 \%$ & $100 \%$ & $0 \%$ & 1 \\
\hline 11 & $90 \%$ & $10 \%$ & $70 \%$ & $30 \%$ & 1 \\
\hline 12 & $90 \%$ & $10 \%$ & $80 \%$ & $20 \%$ & 0.9 \\
\hline 13 & $100 \%$ & $0 \%$ & $100 \%$ & $0 \%$ & 1 \\
\hline 14 & $100 \%$ & $0 \%$ & $100 \%$ & $0 \%$ & 1 \\
\hline 15 & $100 \%$ & $0 \%$ & $40 \%$ & $60 \%$ & 1 \\
\hline 16 & $100 \%$ & $0 \%$ & $40 \%$ & $60 \%$ & 1 \\
\hline 17 & $100 \%$ & $0 \%$ & $70 \%$ & $30 \%$ & 1 \\
\hline 18 & $100 \%$ & $0 \%$ & $100 \%$ & $0,00 \%$ & 1 \\
\hline 19 & $100 \%$ & $0 \%$ & $50 \%$ & $50 \%$ & 1 \\
\hline 20 & $90 \%$ & $10 \%$ & $100 \%$ & $0 \%$ & 1 \\
\hline 21 & $80 \%$ & $20 \%$ & $100 \%$ & $0 \%$ & 0.9 \\
\hline 22 & $100 \%$ & $0 \%$ & $100 \%$ & $0 \%$ & 0.9 \\
\hline 23 & $80 \%$ & $20 \%$ & $60 \%$ & $40 \%$ & 0.9 \\
\hline 24 & $70 \%$ & $30 \%$ & $80 \%$ & $20 \%$ & 0.8 \\
\hline 25 & $90 \%$ & $10 \%$ & $90 \%$ & $10 \%$ & 0.9 \\
\hline 26 & $90 \%$ & $10 \%$ & $80 \%$ & $20 \%$ & 0.9 \\
\hline CVI-Ave & & & & & 0.94 \\
\hline
\end{tabular}


items and response options on the PFV, in terms of clarity and relevance (Table 3 ). They suggested some modifications to the questions about inappropriate compensatory behaviors (items 15 to 19). The group proposed removing the expression in Portuguese "dose recomendada," because it could suggest that there is a recommended dose of medications to avoid weight gain. However, we decided to retain the expression, because it is related to measurement of quantities of medications taken. In common with the patients' evaluation, some of the experts $(40 \%)$ considered item 23 (about SBE) unclear. After discussion with the experts, we decided to maintain the items related to SBE as they were, because these items are not used to diagnose BED. They were included in the original instrument for research purposes only. It is important to take into account that only items 8 and 9 (about binge eating), 10 (binge eating frequency), 11 (associated symptoms during the episode), 13 (distress regarding binge eating), and 14 through 19 (inappropriate compensatory behaviors) are BED diagnosis items. The PFV of the Brazilian QEWP-5 was considered relevant on the basis of its content equivalence. All 26 items were rated with a CVI of 0.80 or higher. Additionally, the CVI-Ave was 0.94.

Participants took a mean time of 12 minutes to answer the questionnaire. In general, the PFV was well evaluated. Therefore, we did not make changes to the final version. The layout of the original version was maintained. The instrument was given the Portuguese name "Questionário sobre Padrões de Alimentação e Peso-5 (QEWP-5)" (see online-only supplementary material).

\section{Discussion}

The QEWP- $5^{13}$ is an updated version (based on DSM$5)^{1}$ of a widely-used self-report instrument (QEWP-R $)^{17}$ for BED screening. This article describes the translation and cross-cultural adaptation of the QEWP-5 into Brazilian Portuguese. To our knowledge, this is the first cross-cultural adaptation of this instrument. The process followed internationally accepted standards, comprising the stages forward translation, comparison of translations and synthesis version, blinded backtranslations, comparison of the back translations with the original version, and an evaluation of comprehensibility. The Brazilian Portuguese version of the QEWP-5 was successfully cross-culturally adapted for future validation and application in Brazil.

The process of cross-cultural adaptation of instruments needs to follow rigorous and standardized guidelines to generate a reliable translated instrument. ${ }^{14}$ This is an essential procedure that enables comparison of results obtained from samples with different cultural backgrounds. ${ }^{18}$ Although there is no consensus on the best methodological approach, international guidelines on this process do agree that symmetrical translation should be conducted, following a "road map" comprising forward translations, back translations, experts' panel, and pre-testing. ${ }^{14,19,20}$

Unlike the QEWP-R, ${ }^{17}$ which was based on the DSMIV-TR, ${ }^{21}$ the QEWP- $5^{13}$ contains the current diagnostic criteria for BED. The major change made to the BED criteria in the DSM- 5 was related to the minimum average frequency of binge eating required for diagnosis. ${ }^{1}$ Thus, the QEWP-5 incorporates the DSM-5 frequency threshold of "at least one binge eating episode per week over the last 3 months, ${ }^{11}$ rather than the DSMIV-TR ${ }^{21}$ criterion of "at least two binge days a week for 6 months."11 Another change made in the QEWP-5 was to alter the threshold for inappropriate compensatory behaviors. In the QEWP-R, ${ }^{17}$ the threshold for misuse in terms of compensatory behaviors was "taking more than twice the recommended dose of medications to avoid weight gain." In contrast, in the QEWP- $5,{ }^{13}$ taking more than the recommended dose of diuretics, obesity drugs, or laxatives is considered misuse.

Another important change in the QEWP-5 was inclusion of questions to assess SBE. SBE describes episodes in which eating is out of control, but the amount of food is not considered unusually large. ${ }^{6}$ There is evidence showing that SBE can cause marked distress and impairment to individuals who experience it, similar to OBE. ${ }^{22}$ The 11 th edition of the International Classification of Diseases for Mortality and Morbidity Statistics (ICD-11) therefore included both OBE and SBE in the diagnostic criteria for BED. ${ }^{23,24}$ Therefore, the QEWP- 5 can also potentially be used to assess BED according to ICD-11 criteria.

It is important to highlight that the main doubts raised in the comprehensibility test were related to items assessing SBE. One possible explanation is that the expression "an amount of food not considered unusually large" is ambiguous. We therefore consider that the problem lies in the definition of SBE itself and not specifically with the question asked in the QEWP-5. Along the same lines, Mitchell et al. ${ }^{25}$ have commented that it is difficult to distinguish OBE from $S B E$ in individuals with BED, especially when self-report instruments (like the QEWP-5) are used. The level of agreement between self-report instruments and clinical interviews for assessment of OBE and SBE tends to be low. ${ }^{25}$ In a study that compared the EDE interview with the EDE-Q for assessment of the features of eating 
disorders in patients with BED, Grilo et al. ${ }^{26}$ found that SBE frequencies assessed with the EDE and the EDE-Q were not significantly correlated and that the magnitude of the difference between them was large. These authors concluded that the EDE-Q may therefore underestimate SBE frequency. ${ }^{26}$

The present study has some limitations. First, although the sample size analyzed for the comprehensibility test was that recommended by the guideline followed when conducting the cross-cultural adaptation, ${ }^{14}$ it could nevertheless be considered too small for generalization of the findings. Second, the fact that all participants in the comprehensibility test were patients from an outpatient eating disorder service limits generalization of results to individuals with other characteristics.

Diagnosing BED is challenging. Several aspects related to binge eating episodes, such as the amount of food eaten, the presence of loss of control over eating and the frequency of the episodes, among others, are sometimes difficult to capture for non-specialists in eating disorders. Unfortunately, in Brazil only the previous version of QEWP (the QEWP-R) is currently available, ${ }^{12}$ which does not include the most recent changes in the DSM- $5^{1}$ criteria, and also the BES, ${ }^{8}$ which is not appropriate for categorical diagnosis. This Portuguese version of the QEWP-5 will therefore be very useful, filling the gap left by lack of an instrument for screening that enables a researcher or clinician to assign a DSM-5 diagnosis of BED and BN.

\section{Conclusion}

The Brazilian Portuguese version of the QEWP5 was correctly adapted. Items were well understood by the target population. This version is available for Brazilian research and clinical settings. The instrument's psychometric properties should be assessed in clinical and non-clinical settings in the next steps of its application.

\section{Acknowledgements}

José Carlos Appolinário has received research grants from Conselho Nacional de Desenvolvimento Científico e Tecnológico (CNPq). The authors are grateful to João Hiluy, Bruno Palazzo Nazar, Marcelo Papelbaum, Maria Francisca Mauro, Arnaldo Cascardo, Maene Cristine, Livia Menescal, Adriana Daquer and Amanda Rodrigues for their assistance in the process of translation of the QEWP-5.

\section{Disclosure}

Marsha D. Marcus is a member of the Scientific Advisory Board of WW International, Inc. José Carlos Appolinário has received research grants, consultancy fees, and advisory board fees from Shire Pharmaceuticals. No other conflicts of interest declared concerning the publication of this article.

\section{References}

1. American Psychiatric Association. Diagnostic and Statistical Manual of Mental Disorders, Fifth Edition (DSM-5). Arlington: American Psychiatric Publishing; 2013.

2. Dahlgren $C L$, Wisting $L, R \varnothing \varnothing$. Feeding and eating disorders in the DSM-5 era: a systematic review of prevalence rates in nonclinical male and female samples. J Eat Disord. 2017;5:1-10.

3. Kessler RC, Berglund PA, Chiu WT, Deitz AC, Hudson JI, Shahly $\mathrm{V}$, et al. The prevalence and correlates of binge eating. Biol Psychiatry. 2013;73:904-14.

4. Stunkard AJ, Allison KC. Binge eating disorder: disorder or marker? Int J Eat Disord. 2003;34 Suppl:S107-16.

5. Fairburn C, Beglin S. Assessment of eating disorders: interview or self-report questionnaire? Int J Eat Disord. 1994;16:363-70.

6. Fairburn $C$, Cooper $Z$. The eating disorder examination. In: Fairburn C, Wilson G, editors. Binge eating: nature, assessment and treatment. New York: Guilford Press; 1993. p. 317-60.

7. Gormally J, Black S, Daston S, Rardin D. The assessment of binge eating severity among obese persons. Addict Behav. 1982;7:4755

8. Freitas S, Lopes CS, Coutinho W, Appolinario JC. Tradução e adaptação para o português da Escala de Compulsão Alimentar Periódica. Braz J Psychiatry. 2001;23:215-20.

9. Freitas SR, Lopes CS, Appolinario C, Coutinho W. The assessment of binge eating disorder in obese women: a comparison of the binge eating scale with the structured clinical interview for the DSM-IV. 2006; 7:282-9.

10. Spitzer RL, Yanovski SZ, Marcus MD. Questionnaire on Eating and Weight Patterns, Revised. Pittsburgh: Behavioral Measurement Database Services; 1994.

11. American Psychiatric Association. Diagnostic and Statistical Manual of Mental Disorders. Fourth Edition (DSM-IV). Washington: American Psychiatric Association; 1994.

12. Borges MBF, Morgan CM, Claudino AM, Da Silveira DX. Validation of the Portuguese version of the Questionnaire on Eating and Weight Patterns - Revised (QEWP-R) for the screening of binge eating disorder. Braz J Psychiatry. 2005;27:319-22.

13. Yanovski SZ, Marcus MD, Wadden TA, Walsh BT. The Questionnaire on Eating and Weight Patterns-5: an updated screening instrument for binge eating disorder. Int J Eat Disord. 2015;48:259-61.

14. Sousa VD, Rojjanasrirat W. Translation, adaptation and validation of instruments or scales for use in cross-cultural health care research: a clear and user-friendly guideline. J Eval Clin Pract. 2011;17:268-74.

15. Lynn MR. Determination and quantification of content validity. Nurs Res. 1986;35:382-5.

16. Waltz CF, Strickland OL, Lenz ER. Measurement in nursing and health research. 3rd ed. New York: Springer; 2005.

17. Yanovski SZ. Binge eating disorder: current knowledge and future directions. Obes Res. 1993;1:306-24.

18. Reichenheim ME, Moraes CL. Operacionalização de adaptação transcultural de instrumentos de aferição usados em epidemiologia. Rev Saude Publica. 2007;41:665-73.

19. Borsa JC, Damasio BF, Bandeira DR. Adaptação e validação de instrumentos psicológicos entre culturas: algumas considerações. Paideia. 2012;22:423-32.

20. Gorenstein C, Wang Y-P, Hungerbühler I. Instrumentos de avaliação em saúde mental. Porto Alegre: Artmed; 2016.

21. American Psychiatric Association. Diagnostic and Statistical Manual of Mental Disorders, Fourth Edition, Text Revision (DSMIV-TR). Washington: American Psychiatric Association; 2000. 
22. Latner JD, Clyne C. The diagnostic validity of the criteria for binge eating disorder. Int J Eat Disord. 2008;41:1-14.

23. First MB, Reed GM, Hyman SE, Saxena S. The development of the ICD-11 clinical descriptions and diagnostic guidelines for mental and behavioural disorders. World Psychiatry. 2015;14:82-90.

24. Reed GM, First MB, Elena Medina-Mora M, Gureje O, Pike KM, Saxena S. Draft diagnostic guidelines for ICD-11 mental and behavioural disorders available for review and comment. World Psychiatry. 2016;15:112-3.

25. Mitchell JE, Devlin MJ, Zwaan M De, Crow SJ, Peterson CB. Diagnosis and epidemiology of binge-eating disorder. In: Mitchell JE, Devlin MJ, Zwaan M De, Crow SJ, Peterson CB, editors. Bingeeating disorder: clinical foudations and treatment. New York: Guilford; 2008. p. 3-86.
26. Grilo CM, Masheb RM, Wilson GT. Different methods for assessing the features of eating disorders in patients with binge eating disorder: a replication. Obes Res. 2001;9:418-22.

\section{Correspondence:}

Carlos Eduardo Ferreira de Moraes

Grupo de Obesidade e Transtornos Alimentares (GOTA)

Instituto de Psiquiatria (IPUB), Universidade Federal do Rio de Janeiro

Av. Venceslau Brás, 71, Campus Praia Vermelha, Botafogo 22290-140 - Rio de Janeiro, RJ - Brazil Tel: +55 (21) 995663661

E-mail: carloseduardofm09@gmail.com 